28 Research Square

\title{
Are Need for Affect and Cognition Culture-Dependent? Implications for Global Public health Campaigns: A Cross-Sectional Study
}

\author{
Min Zhang \\ Bei Zhu \\ Songjiang Jiuting Community Health Center \\ Chunlan Yuan \\ Songjiang Jiuting Community Health Center \\ Chao Chao \\ Fudan University National clinical research center for aging and medicine \\ Jiaofeng Wang \\ Huadong Hospital Affiliated to Fudan University \\ Qingwei Ruan \\ Huadong Hospital Affiliated to Fudan University \\ Zhijun Bao \\ Huadong Hospital Affiliated to Fudan University \\ Jie Chen ( $\square$ laughchen@126.com) \\ https://orcid.org/0000-0003-2691-6677 \\ Kevin (Vin) Arceneaux \\ Temple university Department of Political Science \\ Ryan Vander Wielen \\ Temple University Department of Political Science \\ Greg Jeremy Siegle \\ University of Pittsburgh Department of Psychiatry
}

Shanghai Key Laboratory of Clinical Geriatric Medicine Huadong Hospital Affiliated to Fudan University https://orcid.org/0000-0002-1333-2667

Research article

Keywords: Intrinsic motivation, Need for Affect, Need for Cognition, Cultural differences, Global public health campaign

Posted Date: October 6th, 2020

DOI: https://doi.org/10.21203/rs.3.rs-76618/v1

License: @ (i) This work is licensed under a Creative Commons Attribution 4.0 International License. Read Full License

Version of Record: A version of this preprint was published at BMC Public Health on April 9th, 2021. See the published version at https://doi.org/10.1186/s12889-021-10689-w. 


\section{Abstract}

Background: Cultural differences in affective and cognitive intrinsic motivations could introduce challenges to global public health campaigns, which use cognitive or affective goals to evoke desired attitudes and proactive health-promoting actions. This study aimed to demonstrate cross-cultural differences in affective and cognitive intrinsic motivations, and discuss the potential value of this information in public health promotion.

Methods: A cross-sectional survey, using cross-culturally validated need for affect (NFA) and need for cognition (NFC) scales, was carried out among 1166 Chinese participants and compared to published data from 980 American participants. Additionally, we assessed a highly prevalent symbolic geriatric health condition, hearing loss, in 500 community-dwelling seniors. MANOVA test and Hedge's g statistic were employed to compare the NFA and NFC levels between individuals from different countries and between seniors with and without hearing loss. The relation of early healthcare seeking intention to NFA and NFC was also explored.

Results: The primary Chinese sample demonstrated decreased NFA and NFC in contrast to their American peers. This difference was preserved in the senior sample. Moreover, seniors with hearing loss had even lower NFA and NFC than those without hearing loss. Intention for early healthcare seeking was low but was associated with intrinsic motivation.

Conclusions: There was a general lack of affective and cognitive intrinsic motivation in Chinese individuals, particularly in seniors with hearing loss, compared with their American peers. These differences, point to a potential challenge in framing effective messages for some cultures in the geriatric public health domain. Ideally, recognizing and understanding this challenge will inspire consideration of novel persuasive strategies for these audiences.

\section{Background}

Many disciplines rely on persuasive communication with target audiences around affective goals (e.g., "do X to be happier") or cognitive goals (e.g., "you will think more clearly"). An implicit assumption is that such affective goals are universal. Rather, affective and cognitive orientation (indexed by Need for Affect and Need for Cognition) differs across individuals and is associated with the effectiveness of messages in persuasive communication $(1,2)$ and consequent proactive behaviors ${ }^{(3)}$. Affective and cognitive orientation may be particularly important for global persuasive communication efforts, e.g., public health campaigns, which might yield diverse outcomes across national cultures given similar campaign contents if there are crosscultural differences in affective and cognitive orientation.

Affective and cognitive orientations are indexed by the self-report questionnaires. The Need for Affect (NFA) questionnaire assesses the tendency to approach or avoid emotion-inducing situations and activities ${ }^{(4)}$. People high in NFA tend to rely upon emotional information in attitude formation and the regulation of behavior ${ }^{(1,2)}$. The Need for Cognition (NFC) questionnaire assesses the tendency to engage in and enjoy effortful thinking ${ }^{(5)}$, and is related to rational decision-making ${ }^{(6)}$ and effort expenditure ${ }^{(7)}$. Higher levels of NFA is associated with greater persuasion in response to an affectbased (but not cognition-based) persuasive message, whereas higher levels of NFC is associated with greater persuasion in response to a cognitionbased (but not affect-based) persuasive message ${ }^{(1)}$.

Geriatric health campaign messages are often geared towards affective goals (e.g., happiness, enjoyment) as evidence showed that affective outcome expectancies have significantly more positive impact on older people's intentions to take proactive actions than rationale outcome expectancies ${ }^{(8)}$. However, cultural context may play a role in structuring affective goals. There are important differences across cultures in terms of norms regarding human engagement with emotions ${ }^{(9)}$. East Asian people - especially Chinese - differ from their Western counterparts in affective dispositions ${ }^{(10)}$. For example, a unique study suggested that residents of America who were of Chinese descent tended to value high-arousal positive affect (e.g., excitement) less than Americans of specifically European descent ${ }^{(11)}$; they tended to want to maximize positive and minimize negative affect less than did Americans of European descent and therefore were more likely to feel the bad with the good referred as mixed affective experience

(12). Chinese individuals are also more affected by potential losses in their risk perception than individuals from the Netherlands and the USA ${ }^{(13)}$. Compared to Americans, east Asians are less inclined to overestimate the emotional consequences of future events ${ }^{(14)}$. There are also differences across cultures on people's engagement in thinking. Confucian, Taoist and Buddhist philosophic traditions oppose public argumentation and debate (15), and focus on learning in a mechanical way without thought or meaning, which has evolved to the extent that people in such cultures are considered to lack abstract and critical thinking abilities, to over-emphasize concrete examples, and to lack originality and creativity ${ }^{(16)}$.

Overall, these data suggest that the baseline levels of affective and cognitive intrinsic motivation may decline in certain cultures, so the conventional emotion/cognition-based messaging is likely to be less effective. A prototypical example of diverse outcomes across cultures, given similar campaign contents and service accessibilities, is in the hearing healthcare domain. There are cross-cultural public health outreach efforts spanning China and America. The prevalence of hearing loss in elderly is $45 \sim 63 \%$ in both countries ${ }^{(17)}$. Although advocating early hearing intervention to reduce long term adverse effects of hearing loss has been running for over 2 decades using the same WHO messages, the hearing intervention rate in geriatrics remains low at $<2 \%$ in China ${ }^{(18)}$, whereas it is around $16 \%$ in America ${ }^{(19)}$. Potentially, understanding differences in audiences' affective and cognitive intrinsic motivation at a national level could help health professionals to culture-tailor persuasive messages to particular populations, and hence facilitate successful campaigns global wise. 
The current study examines whether NFA and NFC differ systematically across relatively large Chinese and American samples. Objectives are to 1) develop a culture-fair assessment of NFA by validating the Chinese translated version of Appel et al.'s (20) NFA scale; 2) examine differences on NFA and NFC between a Chinese and American general public samples; 3 ) taking hearing loss as an example, explore whether communitive-based seniors with a public health condition have reasonably high need for affect or cognition as assumed by public health campaigns; and examine whether their intention of early healthcare seeking would relate to NFA and NFC. We hypothesized that the Chinese sample would have different levels of NFA and NFC, compared to the American sample. We also hypothesized that seniors with hearing loss would be similar with those with normal hearing in NFA and NFC as no evidence has shown that hearing loss would alter individuals' affective and cognitive intrinsic motivations. The health-related proactive active intention in seniors is correlated with NFA and NFC.

\section{Methods \\ Participants}

We recruited 1195 Chinese adults to participate in the study from April to July in 2019. This sample size was comparable to the original NFA developing studies ${ }^{(4,20)}$. Of the 1195 samples, 695 (age $=30.3 \pm 13.3 \mathrm{yrs}, 65.6 \%$ female) were recruited through an online survey tool (TengXun Survey), who completed a web-based survey in return for an instant and direct deposit of RMB®2 RMB (USD\$0.3) into their WeChat payment account. Five hundred older participants (age $=66.7 \pm 11.0 \mathrm{yrs}, 64.0 \%$ female) were recruited offline at a local community health center during the annual mandatory physical examination. The community-based participants completed hearing assessments in addition to the NFA and NFC surveys. They were reimbursed $₫ 20$ RMB for their participation and travel. Our American sample was from Arceneaux and Vander Wielen (21). The 1006 individuals in their study completed a web-based survey through Qualtrics Internet in return for "online currency" that can be redeemed for consumer products or cash. Twenty-nine Chinese participants and twenty-six American participants had the same value across the NFA items. Their data were excluded from primary analyses.

\section{Instruments}

\section{English Need for Affect Scale}

The English 10-item short version consists of approach and avoidance subscales with 5 items in each (20). The scale uses a 7-point Likert Scale for responses $[-3=$ Strongly disagree to $3=$ Strongly agree with $0=$ Uncertain]. Some subsequent studies, including Arceneux and Vander Wielen $(21)$, have used a 5-point Likert Scale for responses ( $1=$ Strongly disagree, $5=$ Strongly agree).

\section{Chinese NFA Scale construction}

The Chinese NFA version (see Appendix) was rigorously constructed in line with state-of-the-art standards in cross-cultural research involving translation-bask translation method. The detailed constructing process is provided in the supplemental materials.

\section{Need for Cognition Scale}

Cacioppo et al.'s (5) 18-item scale was used to assess the NFC in the American sample. The Chinese NFC scale validated by Kuang, Shi (22) was used. Hearing assessment

Two hearing-related assessments were administered to the community-based seniors: 1) a simplified pure tone examination (23) using an audiometer (Interacoustics, MA52) and the 10-item Hearing Handicap Inventory for the Elderly-Screening questionnaire ${ }^{(24)}$, these screening tests were used to estimate hearing loss severities; 2) a multiple choice intention question "When do you think you will consider wearing hearing aids and take serious action?" The five choices were: $₫$ When if I have a mild hearing loss; $₫$ When if I have a moderate hearing loss; $₫$ When if I have a severe hearing loss and have difficulty communicating with others; $\varangle$ When if I lose all my hearing; $邓$ I will never consider wearing hearing aids, because they are not helpful.

\section{Procedures}

Chinese participants responded to the Chinese NFA questionnaire developed in the present study (7-point Likert scale for responses) and the Chinese NFC scale. The 500 community-based Chinese seniors completed the hearing assessments additionally. Forty-seven of the 500 participants completed the battery twice with an interval of $3 \sim 4$ weeks; on each occasion, they completed the NFA, NFC, and the one intention question about early hearing care seeking. American participants in Arceneaux and Vander Wielen's ${ }^{(21)}$ study responded to the English NFA and NFC questionnaires.

\section{Analysis methods}

Only $5.9 \%$ of cases had missing data with a total of $0.25 \%$ missing values in our samples. Missing values were managed via full an Information Maximum Likelihood (FIML) approach. The psychometrics of the Chinese NFA questionnaire were examined in terms of test-retest reliability, internal consistency and Cronbach's alpha. Convergent validity was indexed by the correlation between NFA and NFC ${ }^{(20)}$. The construct validity of NFA in the 
Chinese and American samples was examined via Confirmatory Factor Analysis and the Exploratory Structural Equation Modeling (ESEM) with AMOS 24.0 statistic package. The goodness-of-fit indexes were reported following Hu and Bentler ${ }^{(25)}$ recommendations (see supplement for details).

To test our hypothesis about cultural differences on the NFA, the scores of our samples and that of 4 independent European samples reported by Appel, Gnambs ${ }^{(20)}$ were compared using a meta-analysis approach. Effect sizes (Hedges's g values) and associated $95 \%$ confidence intervals were calculated. Responses to the English 5-point scale NFA from the American sample were linearly transformed into 7-point scale values by using the formula $y=3(x-3) / 2$ to match the scale used in the Chinese and Appel et al.'s ${ }^{(20)}$ European samples. Additionally, we compared the correlation between the two latent factors (approach and avoidance) across these samples via t-tests after transforming correlations to a normal distribution using Fisher's $r$ to $z$ transformation. The difference of NFC between the Chinese and American samples was also tested using Hedges's g value.

To explore whether seniors with a highly prevalent public health problem (i.e., hearing loss) have reasonably high intrinsic motivations as assumed by public health campaigns, we applied MANOVA to examine the differences on the NFA and NFC scores between seniors with and without hearing loss. Moreover, correlation analysis was conducted to explore the relationship between the individuals' NFA, NFC and the tendency of early hearing intervention seeking. These analyses were performed using SW 24 (SPSS/IBM, Chicago, IL).

\section{Results}

\section{Q1. Psychometric characterization of a Chinese version of the NFA}

The 10-item Chinese short scale based on ${ }^{(20)}$ showed a test-retest correlation and internal consistency of $r=.943, p<.001, \mathrm{ICC}=.942, p<.001$, and Cronbach's $a=.829, F(9,10485)=54.580, p<.001$. Consistent with Kuang, Shi ${ }^{22}$, the Chinese Need for Cognition (NFC) scale had an internal consistency above .850 , Cronbach's $a=.866, F(17,19805)=99.992, p<.001$. The correlation between the Chinese NFA and NFC was $.221(p<.001)$, comparable with Appel et al.'s ${ }^{(20)}(r=.170)$ finding in the convergent validity testing of NFA.

The results of the confirmatory factor analysis and ESEM demonstrated a satisfactory fit of 10-item NFA's two-factor model in both Chinse and American samples (Table 1)

Table 1

Goodness-of-fit statistics of using Exploratory Structural Equation Modeling (ESEM) and Confirmatory Factor Analysis (CFA) for the Appel et al.'s 10-item NFA scale in the 4 subsamples. Splitting samples in CFA is recommended by Fokkema and Greiff (2017) to minimize model overfit.

\begin{tabular}{|lllllllllll|}
\hline Model tested & $\lambda^{2}$ & df & $\mathbf{p}$ & CFI & TLI & NFI & RMSEA & AIC & ECVI \\
\hline \multirow{2}{*}{ ESEM } & Chinese subsample 1 & 79.325 & 22 & $<.001$ & 0.975 & 0.95 & 0.967 & 0.067 & 165.325 & 0.285 \\
\cline { 2 - 11 } & Chinese subsample 2 & 90.266 & 22 & $<.001$ & 0.971 & 0.94 & 0.962 & 0.073 & 176.266 & 0.302 \\
\cline { 2 - 11 } & American subsample 1 & 40.86 & 22 & $<.001$ & 0.988 & 0.969 &. .974 & 0.041 & 126.86 & 0.251 \\
\cline { 2 - 11 } & American subsample 2 & 52.681 & 22 & $<.001$ & 0.976 & 0.94 & 0.96 & 0.054 & 138.681 & 0.294 \\
CFA & Chinese subsample 1 & 83.327 & 15 & $<.001$ & 0.971 & 0.912 & 0.965 & 0.089 & 187.327 & 0.323 \\
\cline { 2 - 11 } & Chinese subsample 2 & 86.597 & 15 & $<.001$ & 0.969 & 0.908 & 0.964 & 0.09 & 190.597 & 0.326 \\
\cline { 2 - 10 } & American subsample 1 & 24.259 & 15 & $<.001$ & 0.994 & 0.978 & 0.985 & 0.035 & 124.259 & 0.246 \\
\cline { 2 - 10 } & American subsample 2 & 38.27 & 15 & $<.001$ & 0.982 & 0.933 & 0.971 & 0.057 & 138.27 & 0.293 \\
\hline \multirow{2}{*}{ Criterion for goodness of fit } & & & & $\geq 0.9$ & $\geq 0.9$ & $\geq 0.9$ & $\leq 0.1$ & & \\
\hline
\end{tabular}

The factorial invariance of the NFA structure was tested following a stepwise procedure as in ${ }^{(26)}$. Although the baseline model implying Configural Invariance reported a significant chi-square $\left(\chi^{2}(60)=271.21, p<.01\right)$, other indices pointed to good fit from a practical standpoint $(27)$, i.e., comparative fit index $(\mathrm{CFI})=.972$, normed fit index $(\mathrm{NFI})=.965$, mean square error of approximation (RMSEA) $=.041$. This result implied that the measurement structure with two latent factors was invariant among groups. When all factor loadings were constrained, multigroup analysis showed reasonable fitting, $\chi^{2}(84)=414.489, \mathrm{p}<.01, \mathrm{CFI}=.956, \mathrm{NFI}=.946, \mathrm{RMSEA}=.042$ (see supplement for more detailed invariance test statistics). The above results supported that Appel et al.'s (20) 10-item NFA scale is sufficient as a culture-fair assessment of affective intrinsic motivation.

\section{Q2. Cultural Differences In Nfa And Nfc}

The mean NFA approach scores and avoidance scores were computed and compared between the Chinese sample and American sample, and also contrasted to the 4 independent European samples from Appel et al.'s ${ }^{(20)}$ study (see Fig. 1). As shown in Table 2, the effect sizes in terms of Hedges's $\mathrm{g}$ values and the $95 \%$ Cls revealed that the Chinese general public sample had significantly lower NFA approach scores than the American and European samples, and significantly higher NFA avoidance scores than people in other cultures. Compared to the European samples, the American 
adults had significantly lower NFA approach scores and higher avoidance scores. The Chinese general public had significantly lower NFC than their American peers.

Table 2

The mean, standard deviation, sample size of NFA average subscale scores and NFC total scores in multinational samples, and the effect size indices

\begin{tabular}{|c|c|c|c|c|c|c|c|c|c|c|c|c|c|}
\hline & Sample 1 & Sample 2 & $\begin{array}{l}\text { Mean } \\
1\end{array}$ & $\begin{array}{l}\text { Mean } \\
2\end{array}$ & N 1 & N 2 & SD 1 & SD 2 & $\begin{array}{l}\text { Cohen's } \\
\text { d }\end{array}$ & $\begin{array}{l}\text { Hedges's } \\
\mathrm{g}\end{array}$ & SEg & $\begin{array}{l}95 \% \\
\mathrm{Cl}\end{array}$ & $\begin{array}{l}95 \% \\
\mathrm{Cl}\end{array}$ \\
\hline & & & & & & & & & & & & $\begin{array}{l}\text { Lower } \\
\text { limit }\end{array}$ & $\begin{array}{l}\text { Upper } \\
\text { limit }\end{array}$ \\
\hline \multirow[t]{9}{*}{ Approach } & \multirow[t]{5}{*}{$\begin{array}{l}\text { Chinese } \\
\text { adults }\end{array}$} & $\begin{array}{l}\text { American } \\
\text { adults }\end{array}$ & \multirow[t]{5}{*}{.55} & .91 & \multirow[t]{5}{*}{1186} & 980 & \multirow[t]{5}{*}{1.54} & 0.92 & -0.28 & -0.28 & 0.04 & -0.36 & -0.19 \\
\hline & & $\begin{array}{l}\text { German/Austria } \\
\text { students }\end{array}$ & & 1.28 & & 1160 & & 0.96 & -0.57 & -0.57 & 0.04 & -0.65 & -0.48 \\
\hline & & German Adults & & 1.29 & & 627 & & 0.92 & -0.54 & -0.54 & 0.05 & -0.64 & -0.45 \\
\hline & & Austria Couples & & 1.15 & & 126 & & 1.07 & -0.40 & -0.40 & 0.09 & -0.58 & -0.22 \\
\hline & & UK Adults & & 1.02 & & 236 & & 1 & -0.32 & -0.32 & 0.07 & -0.46 & -0.18 \\
\hline & \multirow[t]{4}{*}{$\begin{array}{l}\text { American } \\
\text { adults }\end{array}$} & $\begin{array}{l}\text { German/Austria } \\
\text { students }\end{array}$ & \multirow[t]{4}{*}{.91} & 1.28 & \multirow[t]{4}{*}{980} & 1160 & \multirow[t]{4}{*}{.92} & 0.96 & -0.39 & -0.39 & 0.04 & -0.48 & -0.31 \\
\hline & & German Adults & & 1.29 & & 627 & & 0.92 & -0.41 & -0.41 & 0.05 & -0.51 & -0.31 \\
\hline & & Austria Couples & & 1.15 & & 126 & & 1.07 & -0.26 & -0.26 & 0.09 & -0.44 & -0.07 \\
\hline & & UK Adults & & 1.02 & & 236 & & 1 & -0.12 & -0.12 & 0.07 & -0.26 & 0.02 \\
\hline \multirow[t]{9}{*}{ Avoidance } & \multirow[t]{5}{*}{$\begin{array}{l}\text { Chinese } \\
\text { adults }\end{array}$} & $\begin{array}{l}\text { American } \\
\text { adults }\end{array}$ & \multirow[t]{5}{*}{-.05} & -.91 & \multirow[t]{5}{*}{1186} & 980 & \multirow[t]{5}{*}{1.29} & 1.15 & 0.70 & 0.70 & 0.04 & 0.61 & 0.79 \\
\hline & & $\begin{array}{l}\text { German/Austria } \\
\text { students }\end{array}$ & & -1.39 & & 1160 & & 1.12 & 1.11 & 1.11 & 0.04 & 1.02 & 1.19 \\
\hline & & German Adults & & -1.06 & & 627 & & 1.18 & 0.81 & 0.81 & 0.05 & 0.71 & 0.91 \\
\hline & & Austria Couples & & -1.5 & & 126 & & 1.07 & 1.14 & 1.14 & 0.10 & 0.95 & 1.33 \\
\hline & & UK Adults & & -.55 & & 236 & & 1.2 & 0.39 & 0.39 & 0.07 & 0.25 & 0.53 \\
\hline & \multirow[t]{4}{*}{$\begin{array}{l}\text { American } \\
\text { adults }\end{array}$} & $\begin{array}{l}\text { German/Austria } \\
\text { students }\end{array}$ & \multirow[t]{4}{*}{-.91} & -1.39 & \multirow[t]{4}{*}{980} & 1160 & \multirow[t]{4}{*}{-1.39} & 1.12 & 0.42 & 0.42 & 0.04 & 0.34 & 0.51 \\
\hline & & German Adults & & -1.06 & & 627 & & 1.18 & 0.13 & 0.13 & 0.05 & 0.03 & 0.23 \\
\hline & & Austria Couples & & -1.5 & & 126 & & 1.07 & 0.52 & 0.52 & 0.10 & 0.33 & 0.70 \\
\hline & & UK Adults & & -.55 & & 236 & & 1.2 & -0.31 & -0.31 & 0.07 & -0.45 & -0.17 \\
\hline NFC & Chinese & American & 44.90 & 55.28 & 1186 & 980 & 11.70 & 11.87 & -0.88 & -0.88 & 0.05 & -0.97 & -0.79 \\
\hline
\end{tabular}

The correlation between the NFA approach and avoidance score in the Chinese sample was significantly positive, $r=.44, p<.01$, in contrast to the negative correlations in the American sample $(r=-.30, p<.01), Z=18.08, p<.001$ (see Fig. 2), German/Austria students $(r=-.34, p<.01), Z=19.79, p$ $<.001$, German adults $(r=-.40, p<.01), Z=18.11, p<.001$, Austria couples $(r=-.46, p<.01), Z=10.23, p<.001$, and UK adults $(r=-.44, p<.01), Z=$ $13.18, p<$. 001. There was no significant difference on the approach-avoidance correlation between the American sample and the German/Austria students $(Z=1.03, p=.30)$ and Austria couples $(Z=1.96, p=.05)$ respectively, However, the correlation was significantly smaller in magnitude in the American sample than in the German adults, $Z=2.23, p=.030$, and the UK adults, $Z=2.25, p=.025$.

\section{Q3. Cultural features of NFA and NFC in a population targeted by affective-motivation public health campaigns}

The difference on NFA observed between the Chinese and American general public sample was also present in the older participants ( $\geq 60$ yrs), with significantly lower approach in Chinese seniors $(M=-.57, S D=1.28)$ than American seniors $(M=.93, S D=.81), F(1,586)=167.33, p<.001, \eta_{p}{ }^{2}=.22$, and significant higher avoidance in Chinese seniors $(M=-.42, S D=1.29)$ than American seniors $(M=-.93, S D=1.09), F(1,586)=16.93, p<.001, \eta_{\mathrm{p}}{ }^{2}$ $=.03$.

Hearing loss occurred in $62.6 \%$ of seniors in the current study. As shown in Fig. 3, compared to seniors without hearing loss, those who had hearing loss had significantly lower NFA approach scores, $F(3,482)=9.00, p<.01, \eta_{p}^{2}=.05$, significantly lower avoidance scores, $F(3,482)=26.13, p<.01, \eta_{p}{ }^{2}$ 
$=.05$, and significantly lower NFC scores, $F(3,482)=14.66, p<.01, \eta_{p}{ }^{2}=.08$. There was a small group of individuals who reported having normal hearing but failed the pure tone audiometric test, $n=34$, who had similar levels of NFA and NFC (see Table 3 ) with normal hearing group.

Table 3

The mean and SD of NFA and NFC scores in the Chinese senior participants.

\begin{tabular}{|lllll|}
\hline \multicolumn{5}{|c|}{ Hearing loss } \\
& Normal hearing & Mild hearing loss & Moderate-to-severe hearing loss & Hearing loss deniers \\
\hline NFA approach & $-0.62 \pm 1.02$ & $-1.08 \pm 1.05$ & $-0.94 \pm .88$ & $-0.34 \pm 1.31$ \\
NFA avoidance & $-0.01 \pm 1.16$ & $-0.94 \pm 1.10$ & $-0.93 \pm 1.01$ & $-0.23 \pm 1.27$ \\
\hline NFC & $54.98 \pm 11.94$ & $47.49 \pm 13.54$ & $45.98 \pm 13.94$ & $52.88 \pm 11.60$ \\
\hline
\end{tabular}

Intention to avail themselves of early hearing intervention did not differ between groups, $F(3,482)=1.01, p=.39, \eta_{p}^{2}=.01$. Regardless of hearing status, participants tended to choose "when I have severe hearing loss and have difficulty communicating with others" in response to the question "When do you think you will consider wearing hearing aids and take serious action?" Of the 486 community-based participants, only $6.8 \%$ and $13.8 \%$ reported willingness to seek early intervention when they have mild and moderate hearing loss, respectively. Intention to engage in early hearing care seeking did not significantly correlate with either NFA approach, $r=-.01, p=.89$ or NFA avoidance, $r=.06, p=.22$, but was marginally associated with NFC, $r=.09, p=.04$.

\section{Discussion}

Considering the target audiences' need for affect and cognition in situations involving persuasive communication such as global public health campaigns is important, because matching messages with receivers' affective and cognitive orientation can significantly improve the effectiveness in persuasive communication ${ }^{(1)}$. Cultural differences could be associated with different levels of intrinsic motivation, and introduce challenges to public health campaigns hoping that their messages evoke desired attitudes and proactive health-promoting actions. The current study demonstrated such cultural differences.

We first developed a Chinese translation of the original English Need for Affect scale ${ }^{(20)}$ which demonstrated strong psychometrics. Based on the results of ESEM and the confirmatory factor analysis, we found that Appel et al.'s ${ }^{(20)} 10$-item NFA scale had reliability and cross-cultural validity in the Chinese and American samples, and can thus be used as a culture-fair assessment of NFA.

Our second question was whether there are differences in affective and cognitive intrinsic motivation between the Chinese and American samples. The Chinese sample reported lower motivation to approach emotional events and activities, and a higher tendency to avoid emotion-inducing events and activities than their American and European counterparts (Fig. 1). This finding matched literature suggesting that Chinese participants demonstrate more aversion to strong emotions than Americans ${ }^{(28)}$, and value high-arousal affective states less than Americans ${ }^{(11,12)}$. Cognitive motivation, which indicates less inclination to engage in and enjoy in-depth thinking and process issue-relevant information (29), was also low in the Chinese sample as previously observed ${ }^{(15)}$.

Whereas NFA approach and avoidance were negatively correlated in the American and European samples, they were positively correlated in the Chinese sample. This phenomenon is consistent with observations regarding differential uses of emotion regulation strategies across cultures ${ }^{(30)}$. Cultures that are long-term oriented and value embeddedness and hierarchy (e.g., East Asian), tend to have higher scores on emotion suppression, and the correlation between emotion reappraisal and suppression tend to be positive, whereas Western cultures demonstrate an inverse relationship between affective approach and avoidance. Potentially, Chinese people have higher emotional ambivalence ${ }^{(31)}$, where emotion-desire is often compromised by concerns about the consequences of emotion expression and by efforts to refrain from emotion experience and expression. The goal of these exchanges is to avoid interpersonal conflict and maintain harmony ${ }^{\left({ }^{32}\right)}$, given that Chinese culture places great emphasis on a harmonious and balanced relationship with nature and in social interaction ${ }^{(33)}$. As a result, motivation driven by affect in Chinese may vary along a mixed emotion coping continuum from little approach and little avoidance to strong approach and avoidance (rather than from strong approach to strong avoidance), with a precondition of maintaining balance and harmony of interpersonal and social relationship which is not typical in western cultures. We did not assess other affective constructs, personal traits, or cultural orientation constructs due to the lack of a theoretical motive. Thus factors contributing to cultural differences in NFA remain an open question for further research.

The low correlations between NFC and NFA within samples ( .2) but reliable sample-related differences could also suggest cultural factors are responsible for differences in NFC. The lower levels of NFC in the Chinese sample compared to the American sample could also be driven by the Chinese "harmony first" culture. When people value harmonious social relationships, they tend to decrease effort in information search and deliberation, and in the utilization of issue-relevant information to think, reason and form different attitudes and behaviors from the mainstream (16). Those social strategies are symbols of low cognitive intrinsic motivation and might be a barrier for persuasive communications ${ }^{(34)}$. Alternately, as the NFC is essentially an affective scale assessing how enjoyable cognition is to an individual, given that Chinese generally were only $50 \%$ certain about 
whether approaching/avoiding emotions was enjoyable or not (Fig. 1), it was indicated that Chinese people might benefit less from emotions and therefore are less likely to approach cognition than people in other cultures.

Our third objective was to explore whether the intrinsic motivation level of community-based seniors in China with a specific highly prevalent public health condition (i.e., hearing loss) is high, supporting the idea that affectively motivated health campaigns are warranted. Given the reduction of NFA and NFC in Chinese seniors relative to American seniors, Chinese seniors with hearing loss showed even lower NFA and NFC than those without hearing loss. This result differed from our hypothesis about the similarity of NFA and NFC in seniors with and without hearing loss. The particularly low level of NFA in seniors with hearing loss, compared to those without hearing loss, might be associated with the link between hearing loss and latelife depression ${ }^{(35)}$, the latter of which usually shows motivation disturbance ${ }^{(36)}$. The decreased level of NFC might be explained by Spotts ${ }^{(37)}$ who reported that age-related declines in cognitive ability could affect NFC. Hearing loss is known to associated with cognitive decline in older adults (38), therefore, their cognitive motivations might be constrained by their cognitive capabilities.

The intention of early hearing intervention was low regardless of hearing status; the majority of older people preferred to wait until their hearing condition becomes difficult to cope with and limited intervention benefits are available. Our community-based geriatric sample was a good representation of the hearing health campaign targeting population in China with a hearing loss prevalence of $62.6 \%$, which is consistent with previous report ${ }^{(39)}$. Low levels of NFA and NFC could help to explain why geriatric hearing health campaigns in China are less successful than in America although Chinese campaigns have followed the American model (18). A growing body of research in America has emphasized the importance of affective aspects in efficiently delivering health promotion messages ${ }^{(2)}$. However, perception of affective benefits (e.g., reduction in anxiety, depression and stress, elevation in self-confidence) and/or the instrumental/cognitive outcomes (e.g., reducing the risk of developing severe tinnitus and dementia) assume a matching level of affective and/or cognitive intrinsic motivation from the intended audiences. Our data suggests that this assumption should be questioned.

Our study has multiple limitations. As the original ${ }^{(20)}$ scale scored items $1-7$ and the American version scored items $1-5$, we could not be consistent with both; our version inherited its scale from the Appel, Gnambs ${ }^{(20)}$ sample and was thus inconsistent with how the American version was scored. We addressed this inconsistency by linearly converting responses from the American dataset to a 7-point scale which could have distorted the NFA mean values of the American sample and biased the results. However, this conversion only caused $0.17 \%$ change in the mean value, and was unlikely to account for the $6 \%$ difference between the means on the 7-point scale. For future research reference, we reported the Chinese and American NFA norms by age and sex in both 5- and 7-point scales (see supplement material). The second limitation is the lack of hearing assessment data in the American sample. As a result, we are unable to directly compare the NFA and NFC levels of seniors with hearing loss between the two cultures. In addition, without data on how individuals in any of our samples responded to an actual public health campaign, all our considerations regarding campaigns, at this point, remain speculation; future research is necessary to confirm them.

These limitations notwithstanding, our results demonstrate a general lack of affective and cognitive intrinsic motivation in Chinese individuals, particularly in older adults with hearing loss, compared with the public in American culture. These differences, point to a potential challenge in framing effective messages for some cultures. Ideally, recognizing and understanding this challenge will inspire consideration of novel persuasive strategies for these audiences. For example, using our example of public campaigns for hearing loss, instead of replicating the Western hearing healthcare campaign model, messages could target Asian values more specifically, such as emphasizing a person's ability to be harmonious with nature (e.g., hearing waterfalls) and society (e.g., reduce others' communication burden and stress) rather than individual affective and cognitive goals. Another direction might be strengthening the influence of messages using newly available technology. Breves and Heber ${ }^{(40)}$ reported that people low in NFA were influenced by immersive media while people high in NFA were not, potentially because sensory-rich media experiences offer greater assistance to individuals with lower trait predispositions ${ }^{(41)}$

\section{Conclusions}

We conclude that the Need for Affect (NFA) construct may transcend its Western origins. Assessing NFA and NFC could inform global public health campaigns by helping to frame messages consistent with audiences' culture-dependent intrinsic motivations.

\section{Abbreviations}

NFC, Need for Cognition

NFA, Need for Affect

MANOVA, Multivariate analysis of variance

ESEM, Exploratory Structural Equation Modeling

FIML, Information Maximum Likelihood

CFI, Comparative fit index 
NFI, Normed fit index

RMSEA, mean square error of approximation

\section{Declarations}

\section{Ethics approval and consent to participate}

The study received the approval from the Fudan University Huadong Hospital's Institutional Review Board (No. 2018K154). The community-based participants signed the written consent forms, and the consent to the internet participants was waived.

\section{Consent for publication}

Not applicable.

\section{Competing interests}

The authors declare that they have no competing interests. The audiometric assessment results were discussed with those who were identified as having hearing loss. Information about audiology clinic appointments was provided.

\section{Funding}

The corresponding author disclosed receipt of the following financial support for the research, authorship, and/or publication of this article: This work was supported by the Shanghai Science and Technology Innovation Action Research Fund [\#17411950702].

\section{Authors' contributions}

Contributions of the authors are as follows. Study concept and design: M.Z, G.J.S. Questionnaire development: M.Z, G.J.S, C.Z. Data collection and organization: B.Z, CL,Y, R.V.W, K.A. Statistical analysis and results interpretation:, M.Z, G.J.S. Study supervision: J.C, QW,R., and ZJ, B. Performing of the experiment and Drafting of the manuscript: M.Z, B.Z. All authors reviewed and commented on the manuscript at all stages and approved the final manuscript.

\section{Availability of data and materials}

The datasets used and/or analysed during the current study are available from the corresponding author on reasonable request.

\section{Acknowledgements}

We thank all the participants for their participation in the study. We would like to thank Jess Zhu, Ting Li, Ji Zhang for their comments on the Chinese NFA scale translation. We would also like to thank the nurse practitioners Yue Pan and Wenjuan Lin for their administrative help, including scheduling appointments and organizing the data files.

\section{References}

1. Haddock G, Maio GR, Arnold K, Huskinson T. Should persuasion be affective or cognitive? The moderating effects of need for affect and need for cognition. Pers Soc Psychol Bull. 2008;34(6):769-78.

2. Conner M, Rhodes RE, Morris B, Mceachan R, Lawton R. Changing exercise through targeting affective or cognitive attitudes. Psychol Health. 2011;26(2):133-49.

3. Strauss K, Parker SK, O'Shea D. When does proactivity have a cost? Motivation at work moderates the effects of proactive work behavior on employee job strain. J Vocat Behav. 2017;100:15-26.

4. Maio GR, Esses VM. The need for affect: Individual differences in the motivation to approach or avoid emotions. J Pers. 2001;69(4):583-614.

5. Cacioppo JT, Petty RE. The need for cognition. J Pers Soc Psychol. 1982;42(1):116.

6. Curşeu PL. Need for Cognition and Rationality In Decision-Making. Stud Psychol (Bratisl). 2006;48(2):141-56.

7. Sandra DA, Otto AR. Cognitive capacity limitations and Need for Cognition differentially predict reward-induced cognitive effort expenditure. Cognition. 2018;172:101.

8. Gellert P, Ziegelmann JP, Schwarzer R. Affective and health-related outcome expectancies for physical activity in older adults. Psychol Health. 2012;27(7):816-28.

9. Lim N. Cultural differences in emotion: differences in emotional arousal level between the East and the West. Integrative medicine research. 2016;5(2):105-9.

10. Oyserman D, Coon HM, Kemmelmeier M. Rethinking individualism and collectivism: evaluation of theoretical assumptions and meta-analyses. Psychol Bull. 2002;128(1):3-72. 
11. Tsai JL, Brian K, Fung HH. Cultural variation in affect valuation. J Pers Soc Psychol. 2006;90(2):288-307.

12. Sims T, Tsai JL, Jiang D, Wang Y, Fung HH, Zhang X. Wanting to Maximize the Positive and Minimize the Negative: Implications for Mixed Affective Experience in American and Chinese Contexts. J Pers Soc Psychol. 2015;109(2):292-315.

13. Bontempo RN, Bottom WP, Weber EU. Cross-cultural differences in risk perception: a model-based approach. Risk Anal. 1997;17(4):479-88.

14. Lam KC, Buehler R, Mcfarland C, Ross M, Cheung I. Cultural differences in affective forecasting: the role of focalism. Pers Soc Psychol Bull. 2005;31(9):1296-309.

15. Sanders J, Gass R, Wiseman R, Bruschke J. Ethnic comparison and measurement of argumentativeness, verbal aggressiveness, and need for cognition. Communication Reports. 1992;5(1):50-6.

16. Chan S. The Chinese learner-a question of style. Education + Training. 1999;41(6-7):294-305.

17. Goman AM, Lin FR. Prevalence of Hearing Loss by Severity in the United States. Am J Public Health. 2016;106(10):e1.

18. Zhao F, Manchaiah V, Claire LS, Danermark B, Jones L, Brandreth M, et al. Exploring the influence of culture on hearing help-seeking and hearingaid uptake. Int J Audiol. 2015;54(7):435-43.

19. Chien W, Lin FR. Prevalence of hearing aid use among older adults in the United States. Arch Intern Med. 2012;172(3):292-3.

20. Appel M, Gnambs T, Maio GR. A short measure of the need for affect. J Pers Assess. 2012;94(4):418-26.

21. Arceneaux K, Vander Wielen RJ. The Effects of Need for Cognition and Need for Affect on Partisan Evaluations. Polit Psychol. $2013 ; 34(1): 23-42$.

22. Kuang Y, Shi JQ, Cai YQ. The Chinese Version of Need for Cognition Scale. Chinese Mental Health Journal. 2005;19(1):57-60.

23. Zhang M, Bi ZR, Fu XP, Wang JF, Ruan QW, Zhao C, et al. A parsimonious approach for screening moderate-to-profound hearing loss in a community-dwelling geriatric population based on a decision tree analysis. BMC Genet. 2019;19(214):1-11.

24. Wang G, Li C, Guan W, Xiong J, Kuang S, Hu Y. Development and Evaluation of Reliability and Validity of the Chinese Version of HHIE-S. Journal of Audiology \& Speech Pathology. 2014.

25. Hu LT, Bentler PM. Evaluating model fit. In: RH IH, editor. Structural equation modeling: Concepts, issues, and applications. Thousand Oaks: CA:Sage; 1995. pp. 76-99.

26. Yu L, Shek DTL. Testing factorial invariance across groups: an illustration using AMOS. Int J Disabil Hum Dev. 2014;13(2):205-16.

27. Lt Hu, Bentler PM. Cutoff criteria for fit indexes in covariance structure analysis: Conventional criteria versus new alternatives. Structural equation modeling: a multidisciplinary journal. 1999;6(1):1-55.

28. Eid M, Diener E. Norms for Experiencing Emotions in Different Cultures: Inter- and Intranational Differences. J Pers Soc Psychol. 2001;81(5):86985.

29. Petty RE, Briñol P, Loersch C, McCaslin MJ, Leary MR, Hoyle RH. The need for cognition. Handbook of individual differences in social behavior. 2009:318-29.

30. Matsumoto D, Yoo SH, Nakagawa S. Culture, emotion regulation, and adjustment. J Pers Soc Psychol. 2008;94(6):925-37.

31. Chen SX, Cheung FM, Bond MH, Leung JP. Decomposing the construct of ambivalence over emotional expression in a Chinese cultural context. Eur J Pers. 2005;19(3):185-204.

32. Cheung FM, Leung K, Zhang J-X, Sun H-F, Gan Y-Q, Song W-Z, et al. Indigenous Chinese Personality Constructs: Is the Five-Factor Model Complete? J Cross Cult Psychol. 2001;32(4):407-33.

33. Wang L, Juslin H. The Impact of Chinese Culture on Corporate Social Responsibility. The Harmony Approach. 2009;88(3 Supplement):433-51.

34. Lassiter GD, Briggs MA, Bowman RE. Need for cognition and the perception of ongoing behavior. Pers Soc Psychol Bull. 1991;17(2):156-60.

35. Rutherford BR, Brewster K, Golub JS, Kim AH, Roose SP. Sensation and Psychiatry: Linking Age-Related Hearing Loss to Late-Life Depression and Cognitive Decline. Am J Psychiatry. 2017:appiajp201717040423.

36. Forsell Y, Jorm AF, Winblad B. Association of age, sex, cognitive dysfunction, and disability with major depressive symptoms in an elderly sample. The American journal of psychiatry. 1994.

37. Spotts $\mathrm{H}$. Evidence of a relationship between need for cognition and chronological age: Implications for persuasion in consumer research. ACR North American Advances. 1994.

38. Lin FR, Yaffe K, Xia J, Xue Q-L, Harris TB, Purchase-Helzner E, et al. Hearing loss and cognitive decline in older adults. JAMA internal medicine. 2013;173(4):293-9.

39. Wang Y, Mo L, Li Y, Zheng Z, Yu Q. Analysing use of the Chinese HHIE-S for hearing screening of elderly in a northeastern industrial area of China. International Audiology. 2016;56(4):242-7.

40. Breves P, Heber V. Into the Wild: The Effects of $360^{\circ}$ Immersive Nature Videos on Feelings of Commitment to the Environment. Environmental Communication. 2020;14(3):332-46.

41. Ahn SJ, Bostick J, Ogle E, Nowak KL, McGillicuddy KT, Bailenson JN. Experiencing nature: Embodying animals in immersive virtual environments increases inclusion of nature in self and involvement with nature. Journal of Computer-Mediated Communication. 2016;21(6):399-419.

\section{Figures}

Page $9 / 11$ 


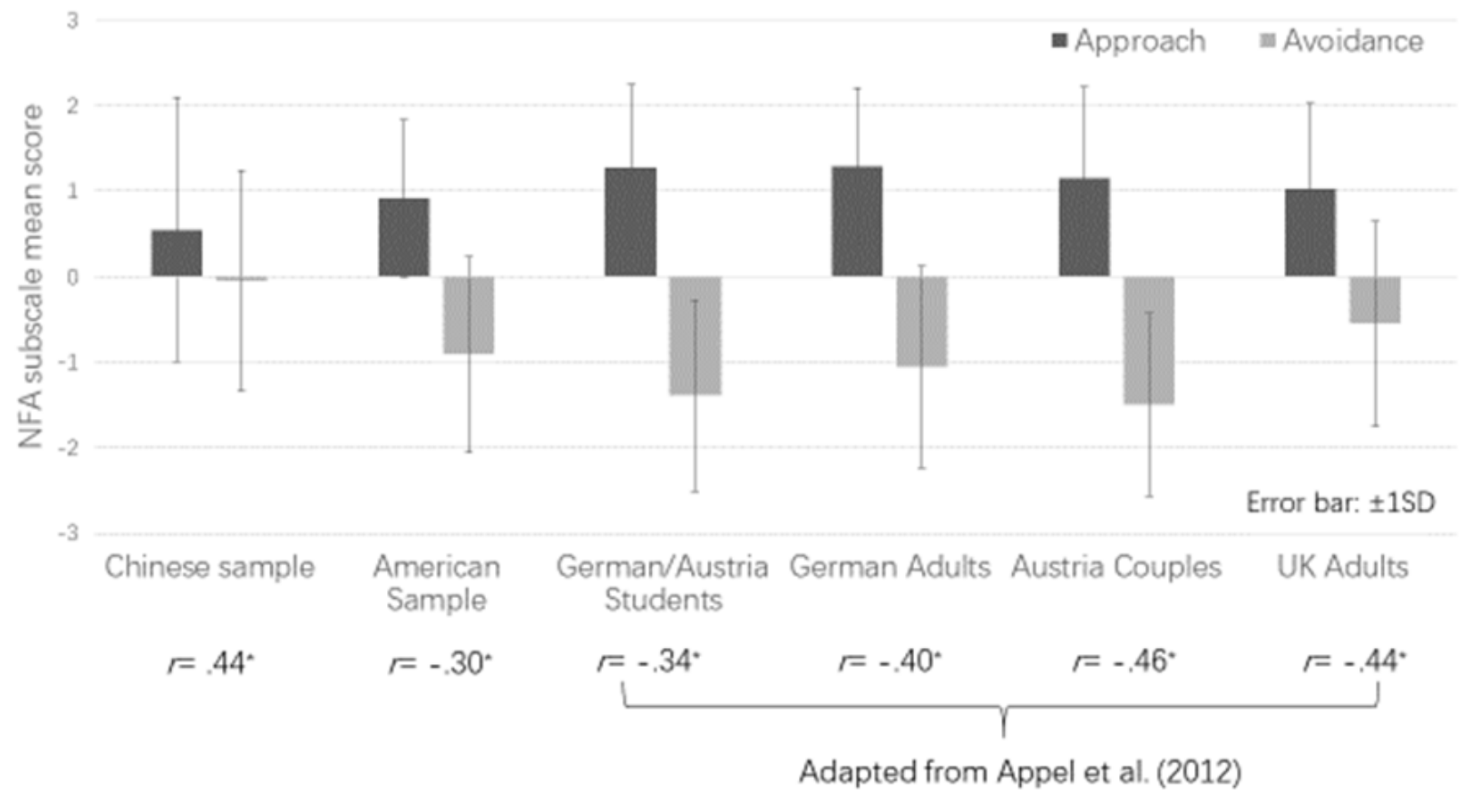

Figure 1

Comparisons of the NFA subscale mean scores across different cultural samples. The r values indicate the correlation between approach and avoidance scores. * indicate a significance at a level of .05.

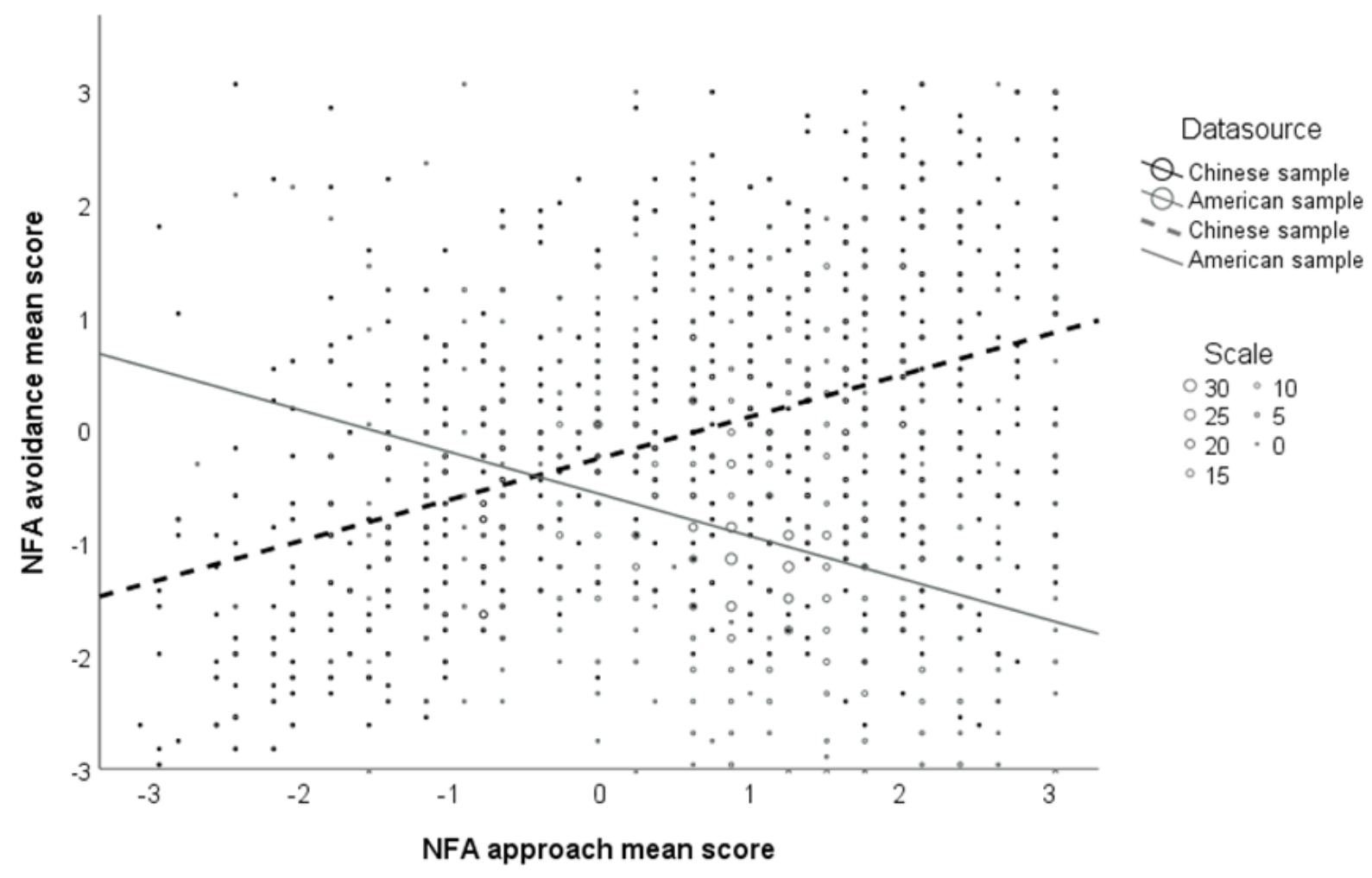

Figure 2

Density plot of NFA approach and avoidance scores of the Chinese and American samples. The fit lines represent the correlation between approach and avoidance scores in each sample. 


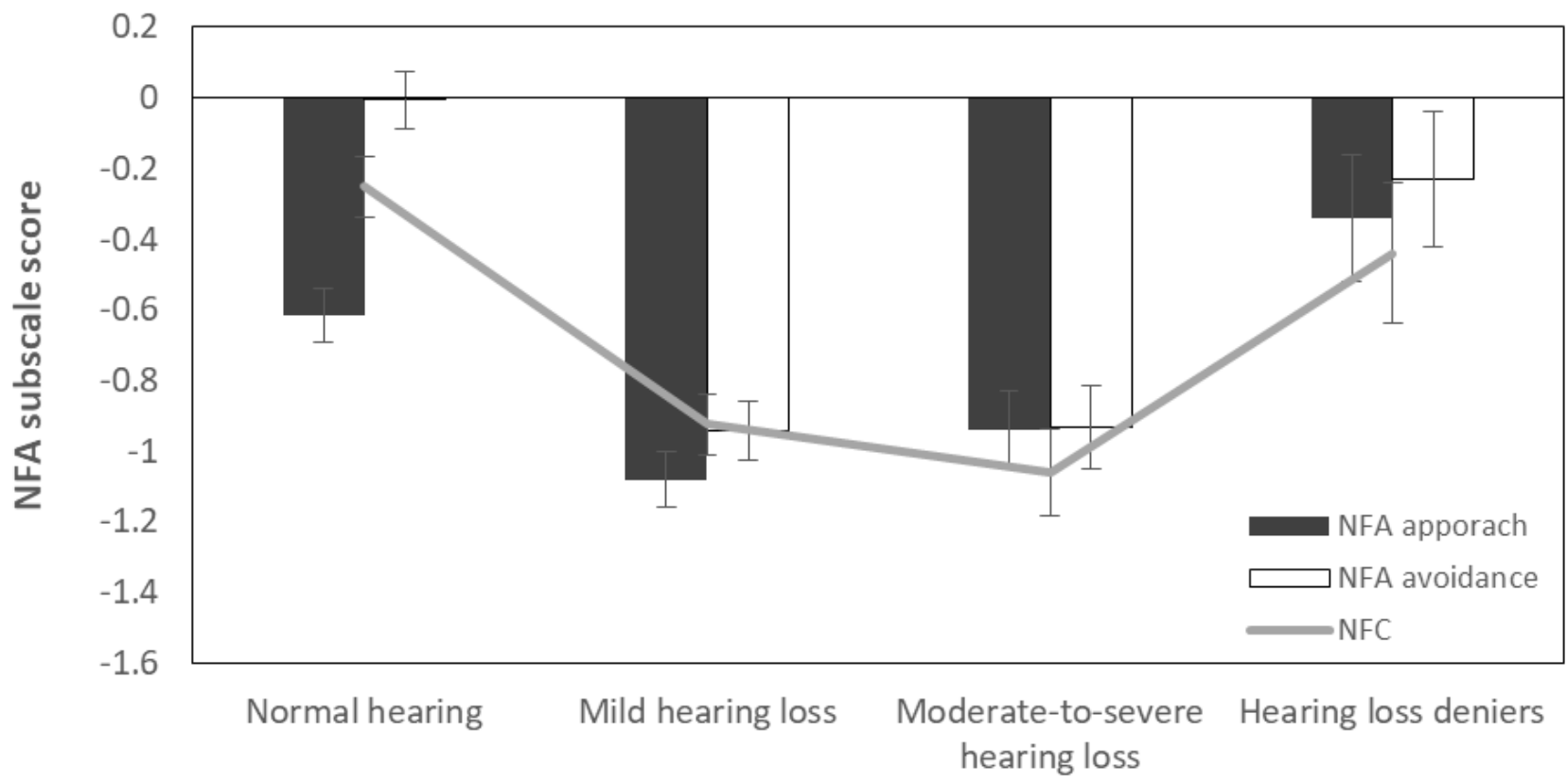

60

58

56

54

52

50 近

48 㟐

46

44

42 40

\section{Hearing status}

\section{Figure 3}

Comparing NFA approach, NFA avoidance and NFC between seniors with and without hearing loss. Error bars represent \pm 1 SE.

\section{Supplementary Files}

This is a list of supplementary files associated with this preprint. Click to download.

- Appendix.docx

- Areneedforaffectandcognitionsupplement.docx 